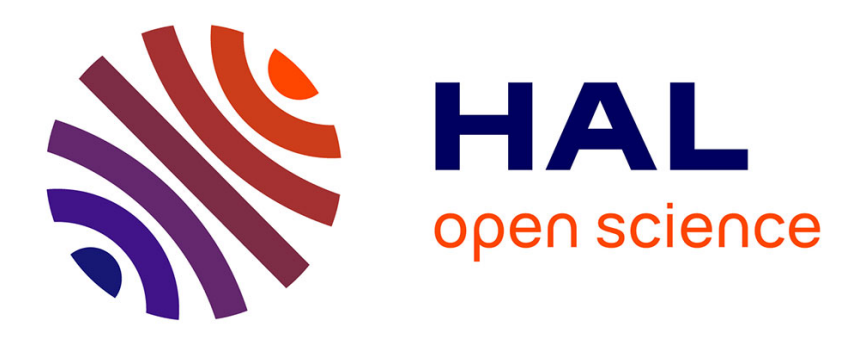

\title{
Deformation retracts to intersections of Whitney stratifications
}

Saurabh Trivedi, David Trotman

\section{To cite this version:}

Saurabh Trivedi, David Trotman. Deformation retracts to intersections of Whitney stratifications. Journal of Singularities, 2020, 22, pp.315-320. 10.5427/jsing.2020.22s . hal-03186950

\section{HAL Id: hal-03186950 \\ https://hal.science/hal-03186950}

Submitted on 1 Apr 2021

HAL is a multi-disciplinary open access archive for the deposit and dissemination of scientific research documents, whether they are published or not. The documents may come from teaching and research institutions in France or abroad, or from public or private research centers.
L'archive ouverte pluridisciplinaire HAL, est destinée au dépôt et à la diffusion de documents scientifiques de niveau recherche, publiés ou non, émanant des établissements d'enseignement et de recherche français ou étrangers, des laboratoires publics ou privés. 


\title{
DEFORMATION RETRACTS TO INTERSECTIONS OF WHITNEY STRATIFICATIONS
}

\author{
SAURABH TRIVEDI \& DAVID TROTMAN
}

\begin{abstract}
We give a counterexample to a conjecture of Eyral on the existence of deformation retracts to intersections of Whitney stratifications embedded in a smooth manifold. We then prove that the conjecture holds if the stratifications are definable in some o-minimal structure without assuming any regularity conditions. Moreover, we also show that the conjecture holds for Whitney stratifications if they intersect transversally.
\end{abstract}

\section{INTRODUCTION}

In 22 Eyral proved the existence of deformation retracts to intersections of Whitney stratifications sitting inside a compact real analytic manifold, and used the result to prove connectivity properties of such intersections. He later used these results to find examples of global rectified homotopical depths and proved a conjecture of Grothendieck on homotopical depth; see [3].

In proving his results Eyral exploits the triangulability properties of compact real analytic manifolds. He then conjectures the existence of deformation retracts to intersections of Whitney stratifications embedded in any non-compact smooth manifold. More precisely, he conjectures the following statement:

Conjecture 5.2 in 2 . Let $M$ be a smooth manifold, $A$ and $B$ be two closed subsets of $M$ and $C$ a closed subset of $B$. Suppose that there exist a Whitney stratification of $B$ adapted to $C$ (i.e. $C$ is a union of strata of $B)$ and a Whitney stratification of $A$ whose strata intersect the strata of $C$ transversally. Then, there exists a neighbourhood $W$ of $A \cap B$ in $B$ such that the couple $(A \cap B,(A \cap B) \backslash(A \cap B \cap C))$ is a strong deformation retract of the couple $(W, W \backslash(W \cap C))$.

He further conjectures that certain pairs of intersection of Whitney stratifications embedded in any smooth manifold (not necessarily compact) are highly connected and claims that this can be proved using the above conjecture; see Conjecture 5.1 in [2].

We show by a simple counterexample that Conjecture 5.2 is false in general.

Date: February 25, 2019. 
Let $M=\mathbb{R}^{2}, B=x$-axis. Let $A$ be the graph of

$$
y= \begin{cases}x^{3} \sin (1 / x) & x \neq 0 \\ 0 & x=0\end{cases}
$$

in $\mathbb{R}^{2}$. Choose $C$ such that $A \cap C$ is empty. Then $A \cap B$ is an infinite (double) sequence of points. Any neighbourhood $W$ of $A \cap B$ will have a component containing the origin and infinitely many points of $A \cap B$. There exists no retraction of $W$ onto $A \cap B$ since the image of a connected set under a continuous map is also connected. Thus $A \cap B$ is not a (strong deformation) retract of any such $W$.

We remark that though the function $y$ above is not smooth, a smooth counterexample can easily be given. For example we can take $A$ to be the graph of

$$
y= \begin{cases}e^{-1 / x^{2}} \sin (1 / x) & x \neq 0 \\ 0 & x=0\end{cases}
$$

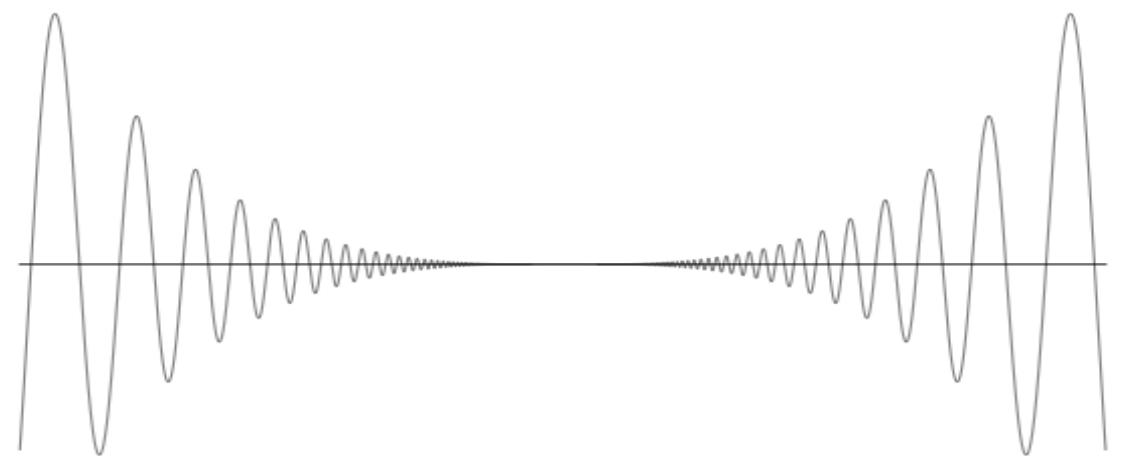

FIGURE 1.

In this article we show that the conjecture holds without the hypothesis of Whitney regularity of stratifications if the strata are assumed to be definable in some o-minimal structure. Furthermore, the conjecture also holds with the extra assumption of transverse intersection of the Whitney stratifications of $A$ and $B$.

\section{TRiangulations of Definable SETS AND Whitney STRATIFICATIONS}

In this section we recall the definition of triangulations and present some of the results about triangulability of stratifications and definable sets in 
o-minimal structures. For definitions of definable sets and o-minimal structures we refer the reader to van den Dries [11. For definitions of stratifications and the regularity conditions $(a)$ and $(b)$ of Whitney we refer to the Ph.D. thesis of the second author [10]. For definitions of simplices, open simplices, simplicial complexes and polytopes we refer to Munkres [7].

Recall that a topological set $X$ is said to be triangulable if there exists a simplicial complex $K$ and a homeomorphism $\phi:|K| \rightarrow X$, where $|K|$ is the polytope of $K$. The simplicial complex $K$ is then said to be a triangulation of $X$. We remark that we allow $K$ to be a simplicial complex with an infinite number of simplices and recall that if $K$ is finite then the polytope $|K|$ is compact and conversely if $A \subset|K|$ is compact, then $A \subset\left|K_{0}\right|$ for some finite subcomplex $K_{0}$ of $K$; see Lemma 2.5 in Munkres [7. This implies that if a triangulable set $X$ is compact then its triangulation is finite. It is well known that any smooth manifold is triangulable; see Part 2 of Munkres [6].

We know that the definable sets in any o-minimal structure can be triangulated. Let us recall the precise statement on triangulations of definable sets; a proof of this result can be found in Coste [1] or van den Dries [11].

In what follows by a definable set we mean a set definable in an o-minimal structure $\mathcal{D}$ over $\mathbb{R}$. Let us mention that definable sets admit definable Whitney stratifications, i.e. every definable set can be stratified into finitely many connected definable submanifolds called strata, such that every pair of adjacent strata satisfies Whitney (b)-regularity; see [8] for a proof.

Theorem 2.1. Let $A \subset \mathbb{R}^{n}$ be a compact definable set and $\left\{B_{1}, \ldots, B_{k}\right\}$ be definable subsets of $A$. Then, there exists a definable homeomorphism $\Phi:|K| \rightarrow A$ from a finite simplicial complex $K$ onto $A$ such that each $B_{i}$ is a union of images of open simplices of $K$ under $\Phi$.

From this it immediately follows that:

Theorem 2.2. Let $\Sigma$ be a definable stratification of a definable subset $V$ of a compact definable set $A$ in $\mathbb{R}^{n}$, then there exists a definable triangulation $\Phi:|K| \rightarrow A$ of $A$ such that every stratum of $\Sigma$ is a union of images of open simplices of $K$ under $\Phi$.

In short, every definable stratification can be triangulated.

Although the results are stated for compact definable subsets of $\mathbb{R}^{n}$, noncompact definable subsets can also be triangulated. This can be seen as follows:

Let $A$ be a definable subset (non-necessarily compact) of $\mathbb{R}^{n}$ and $B_{1}, \ldots, B_{k}$ be definable subsets of $B$. Take the compactification $\mathbb{P}^{n}$ of $\mathbb{R}^{n}$ and consider $A$ as an embedded subset of $\mathbb{P}^{n}$. We know that $A$ considered as an embedded subset of $\mathbb{P}^{n}$ is still a definable subset of some $\mathbb{R}^{N}$ where $\mathbb{P}^{n}$ embeds. We remark here that such an embedding of a non-definable set might not be triangulable, a typical example is the embedding of the graph of $\sin (x)$ in 
$\mathbb{P}^{2}$. We can then apply the above results to $\mathbb{P}^{n}$ and obtain a triangulation of $A$.

Thus, we have:

Theorem 2.3. Let $\Sigma$ be a definable stratification of a definable subset $V$ of a definable set (not necessarily compact) $A$ in $\mathbb{R}^{n}$, then there exists a triangulation $\Phi:|K| \rightarrow A$ of $A$ such that every stratum of $\Sigma$ is a union of images of open simplices of $K$ under $\Phi$.

Furthermore, we know also from Goresky [4] that Whitney stratifications of closed subsets of smooth manifolds are triangulable. We restrict to closed subsets to avoid any pathalogies. Actually, Goresky proved that any abstract prestratified set in the sense of Mather [5] is triangulable. Also, Mather [5] proved thar every Whitney stratified set admits the structure of an abstract stratified set. It follows from this that Whitney stratified sets in $\mathbb{R}^{n}$ are triangulable. Let us mention this result in the following theorem:

Theorem 2.4. Let $M$ be a smooth manifold and $A$ be a closed subset of $M$ admitting a Whitney (b) regular stratification. Then, there exists a simplicial complex $K$ whose polytope is homeomorphic to $M$ and such that every stratum of $A$ is a union of images of open simplicies of $K$ under the homeomorphism.

We give an example of a Whitney (a)-regular stratification which is not triangulable. Consider the set $X$ given by the closure of the graph of $\sin (1 / x)$ for $x>0$ in $\mathbb{R}^{2}$. Stratify it with three strata, the limiting points of the interval in the $y$-axis, the open interval in the $y$-axis and the graph of $\sin (1 / x)$ for $x>0$. This is a Whitney $(a)$-regular stratification, but is not triangulable since it is not path-connected. The set is not a definable set. Furthermore, the intersection $U$ of the $x$-axis, which is a transverse intersection with the set $X$, does not have any open set in the $x$-axis that retracts to the set $U$.

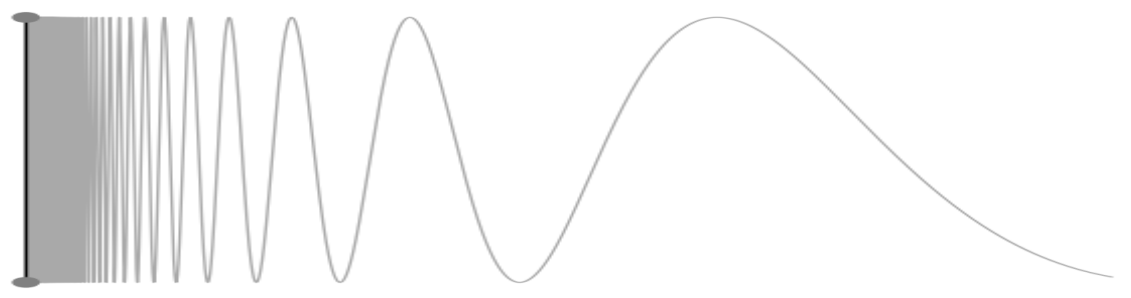

Figure 2.

\section{EXISTENCE OF NeIGHBourhoods OF SUBCOMPLEXES}

In this section we will show how to use barycentric subdivisions of a simplicial complex to obtain neighbourhoods of subcomplexes. These neighbourhoods will be used to construct deformation retracts in the next section. 
The construction of the neighbourhoods is standard but we describe it here for the sake of clarity.

We first of all recall the definition of the 'join' of two simplicial complexes. Let $K_{1}$ and $K_{2}$ be two simplicial complexes. The join of $K_{1}$ and $K_{2}$, denoted by $K_{1} * K_{2}$ is the simplicial complex spanned by the vertices of $K_{1}$ and $K_{2}$ together.

Let $K$ be a simplicial complex and $L$ be a subcomplex of $K$. Denote by $|K|$ the polytope of $K$ and that of $L$ by $|L|$. Let $K^{\prime}$ be the first barycentric subdivision of $K$. Since $L$ is a subcomplex this subdivision induces a subdivision of $L$. Recall that the polytope does not change after the subdivision. Denote by $K \div L$ the subcomplex of $K^{\prime}$ generated by the vertices of $K^{\prime}$ that are not in $L^{\prime}$. Then, there is a natural embedding of $|K|$ onto $|L * K \div L|$. This embedding allows us to write elements of $|K|$ as tuples $(x, t, y)$ where $t \in[0,1], x \in|K \div L|$ and $y \in|L|$. Define

$$
\mathcal{N}(L)=\{p=(x, t, y) \in|K||t \in[0,1), x \in| K \div L \mid \text { and } y \in|L|\} .
$$

It is then easy to see that $\mathcal{N}(L)$ is an open neighbourhood in $|K|$ of $|L|$. For example, see the picture below.

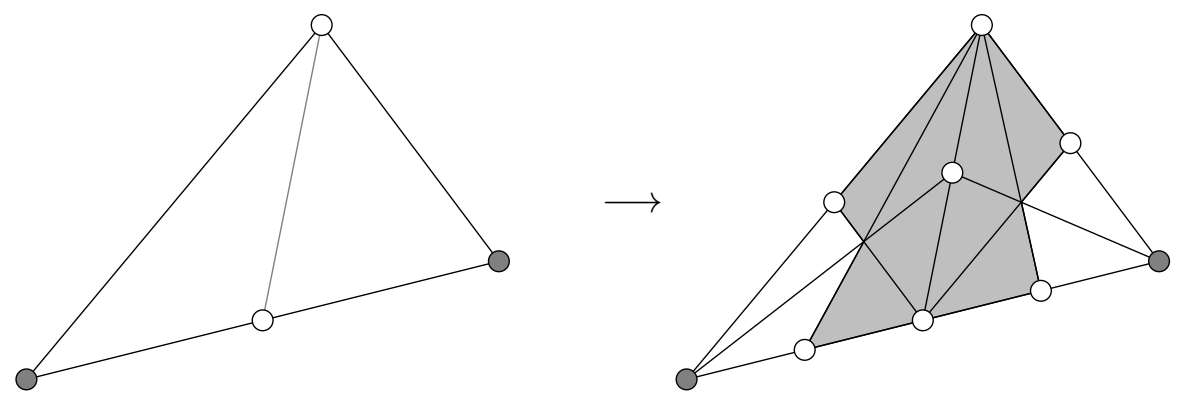

FiguRE 3.

In the picture above the complex $|K|$ is the full triangle while $|L|$ consists of the simplex spanned by the white vertices on the left side of the picture above. The constructed neighbourhood of $|L|$ is the shaded region. It is clear that $|K \div L|$ is what remains after deleting the shaded region.

Moreover, for $t_{0} \in(0,1]$ the set

$$
\mathcal{N}\left(L, t_{0}\right)=\left\{p=(x, t, y) \in|K|\left|t \in\left[0, t_{0}\right), x \in\right| K \div L \mid \text { and } y \in|L|\right\} .
$$

also defines a neighbourhood of $L$ in $K$. By varying $t_{0}$ we get a system of neighbourhoods of $L$ in $K$. Notice that we can also construct neighbourhood of an open subcomplex of $K$. Here, by an open subcomplex we mean a union of open simplices of $K$. More precisely, given an open subcomplex, we can first take the union of its open simplices with their boundaries to 
get a subcomplex of $K$. We can then follow the steps to find a neighbourhood of this subcomplex which also works as a neighbourhood of the open subcomplex we started with.

Finally, if $\phi:|K| \rightarrow X$ is a triangulation of a topological space $X$ such that a subspace $Y \subset X$ is the image of a subcomplex $L$ of $K$ under $\phi$, then the image of $\mathcal{N}\left(L, t_{0}\right)$ under $\phi$ is a neighbourhood of $Y$ in $X$. The closure of $N\left(L, t_{0}\right)$ in $|K|$ is said to be a closed neighbourhood of $L$ in $K$. In the rest of the article, by a neighbourhood $\mathcal{N}(Y)$ of a subspace of a trigulable space $X$ we mean the image of $\mathcal{N}\left(L, t_{0}\right)$ (for some $t_{0} \in[0,1)$ ) under the homeomorphism of the triangulation of $X$. It is then easy to see that $Y$ is a deformation retract of $\mathcal{N}(Y)$. Moreover every neighbourhood of $Y$ in $X$ contains a neighbourhood of type $\mathcal{N}(Y)$; see Proposition 1.4 and 1.5 of Eyral [2].

\section{Construction of Deformation Retracts}

In this section we prove the main results. We first need the following lemma whose proof closely follows the proof of Proposition 1.6 in Eyral [2] and is left to the reader:

Lemma 4.1. Let $X$ be a triangulable space, $Y$ and $Z$ be two subspaces of $X$ that are images of some open simplices of the triangulation of $X$ and $\mathcal{N}(Y)$ the neighbourhood of $Y$ in $X$, then there exists a system of neighbourhoods $\left\{V_{\alpha}\right\}$ of $Y$ in $X$ such that, for every $\alpha,\left(V_{\alpha}, V_{\alpha} \backslash Z\right)$ is a deformation retract of $(\mathcal{N}(Y), \mathcal{N}(Y) \backslash Z)$.

We prove that:

Theorem 4.2. Let $M$ be a definable submanifold of $\mathbb{R}^{n}$ and $A, B$ and $C \subset B$ be closed definable subsets in $M$. Then, there exists a neighbourhood $W$ of $A \cap B$ in $B$ such that the couple $(A \cap B,(A \cap B) \backslash(A \cap B \cap C))$ is a strong deformation retract of the couple $(W, W \backslash(W \cap C))$.

Proof. By Theorem 2.3, we can choose a triangulation $\Phi:|K| \rightarrow M$ of $M$ such that $A$ and $B$ are union of images of some open simplices of $K$. Furthermore, $K$ can be chosen in such a way that $C$ is also a union of the image of open simplices of a sub-complex of the complex $K^{\prime}$ that triangulates $B$, i.e. $\Phi\left(K^{\prime}\right)=B$.

Since finite intersections of definable sets are definable, we can choose a triangulation, a subdivision of $K$ if necessary, of $M$ adapted to $A, B, C$, $A \cap B$ and $A \cap B \cap C$. Moreover, $(A \cap B) \backslash(A \cap B \cap C)$ is also a definable set. Thus, subdividing $K$ if necessary, we can assume that all these definable sets are union of images of some open simplices of $K$ under $\Phi$. Now consider the neighbourhood of $W=\mathcal{N}(A \cap B)$ of $A \cap B$ and constructed in the previous section. By Lemma 4.1, it is then clear that $(A \cap B,(A \cap B) \backslash(A \cap B \cap C))$ is a deformation retract of $(W, W \backslash(W \cap C))$. This concludes the proof of the theorem. 


\section{Moreover,}

Theorem 4.3. Let $M$ be a smooth manifold and $A, B$ and $C \subset B$ be closed subsets of $M$. Suppose there exist a Whitney stratification of $B$ adapted to $C$ (i.e. $C$ is a union of strata of $B$ ) and a Whitney stratification of $A$ whose strata intersect the strata of $B$ transversally. Then, there exists a neighbourhood $W$ of $A \cap B$ in $B$ such that the couple $(A \cap B,(A \cap B) \backslash(A \cap$ $B \cap C))$ is a strong deformation retract to the couple $(W, W \backslash(W \cap C))$.

Proof. By Theorem 2.4, we can choose a triangulation $\Phi:|K| \rightarrow M$ of $M$ such that the strata of $A$ and $B$ are union of images of open simplices of $K$ under $\phi$. Furthermore, $K$ can be chosen in such a way that $C$ is also the image of open simplices of a sub-complex of the complex $K^{\prime}$ that triangulates $B$, i.e. $\Phi\left(K^{\prime}\right)=B$.

Now, since $A$ and $B$ are Whitney stratifications, the intersection $A \cap B$ is also a Whitney stratification; see Orro and Trotman [9]. Thus, we can suitably choose a triangulation, refinement of $K$ if necessary, of $M$ adapted to the strata of $A, B, C, A \cap B$ and $A \cap B \cap C$. That is, every stratum of the five stratifications is a union of images of open simplices of $K$ under $\Phi$. Now consider the neighbourhood $W=\mathcal{N}(A \cap B)$ of $A \cap B$ constructed in the previous section. By Lemma 4.1, it is then clear that $(A \cap B,(A \cap B) \backslash$ $(A \cap B \cap C))$ is a deformation retract of $(W, W \backslash(W \cap C))$. This concludes the proof of the theorem.

\section{REFERENCES}

[1] M. Coste, An Introduction to o-minimal Geometry, Dip. Mat. Univ. Pisa, Dottorato di Ricerca in Matematica, Istituti Editoriali e Poligrafici Internazionali, Pisa, 2000.

[2] C. Eyral, Sur l'homotopie des espaces stratifiés, Internat. Math. Res. Notices (1999), no. $13,717-734$.

[3] _ Profondeur homotopique et conjecture de Grothendieck, Ann. Sci. École Norm. Sup. (4) 33 (2000), no. 6, 823-836.

[4] R. M. Goresky, Triangulation of stratified objects, Proc. Amer. Math. Soc. 72 (1978), no. $1,193-200$.

[5] J. N. Mather, Notes on topological stability, Bull. Amer. Math. Soc. 49 (2012), 475506.

[6] J. R. Munkres, Elementary differential topology, Lectures given at Massachusetts Institute of Technology, Fall, vol. 1961, Princeton University Press, Princeton, N.J., 1963.

[7] Elements of algebraic topology, Addison-Wesley Publishing Company, Menlo Park, CA, 1984.

[8] N. Nguyen, S. Trivedi, and D. J. A. Trotman, A geometric proof of the existence of definable Whitney stratifications, Illinois J. Math. 58 (2014), no. 2, 381-389.

[9] P. Orro and D. Trotman, Regularity of the transverse intersection of two regular stratifications, London Mathematical Society Lecture Note Series, p. 298-304, Cambridge University Press, 2010.

[10] D. J. A. Trotman, Whitney stratifications: Fault and detectors, Ph.D. Thesis, University of Warwick (1977).

[11] L. van den Dries, Tame topology and o-minimal structures., Cambridge: Cambridge University Press, 1998. 
Departamento de Matemática, iCMC - Universidade de São Paulo, 134560970, SÃo Carlos, S.P, Brasil

I2M (UMR 7353), Centre de Mathématiques et Informatique, AiX-Marseille Université, 39 rue Joliot-Curie, 13453 Marseille Cedex 13, France. 